\title{
The X/ $\gamma$-ray correlation in NGC 4945 and the nature of its $\gamma$-ray source
}

\author{
Rafał Wojaczyński* \\ University of Lodz, University of Trieste \& INFN Trieste \\ E-mail: rafal.wojaczynski@wp.pl
}

Andrzej Niedźwiecki

University of Lodz

E-mail: niedzwieckiduni.lodz.pl

\begin{abstract}
We report hints for the correlation between the X-ray and $\gamma$-ray emission in the nearby galaxy NGC 4945, which harbors both an active galactic nucleus and a nuclear starburst region. We have divided the Fermi/LAT observations of NGC 4945 into two datasets, comprising events detected during the low $(\mathrm{L})$ and high $(\mathrm{H})$ level of X-ray emission from the active nucleus of this galaxy, determined using the Swift/BAT light curve. Both datasets contain an equal amount of 3.8 years of LAT data and NGC 4945 is detected with a similar statistical significance of $\sim 15 \sigma$ in L and $14 \sigma$ in $\mathrm{H}$. However, the slope of the $\gamma$-ray spectrum hardens with the increase of the X-ray flux, with the photon index $\Gamma=2.47 \pm 0.07$ in $\mathrm{L}$ and $2.11 \pm 0.08 \mathrm{in} \mathrm{H}$. The change is confirmed by a systematic variation of the spectral energy distribution as well as a substantial reversal of the $\gamma$ ray signal in significance maps for low and high $\gamma$-ray energies. The $\mathrm{X} / \gamma$-ray correlation indicates that the $\gamma$-ray production is dominated by the active nucleus rather than by cosmic rays interacting with the interstellar medium. We discuss possible locations of the $\gamma$-ray source. We also compare NGC 4945 with other starburst galaxies detected by LAT and we note similarities between those with active nuclei, e.g. unlikely high efficiencies of $\gamma$-ray production in starburst scenario, which argues for a significant contribution of their active nuclei to the $\gamma$-ray emission.
\end{abstract}

7th Fermi Symposium 2017

15-20 October 2017

Garmisch-Partenkirchen, Germany

${ }^{*}$ Speaker. 